\title{
Multivalued Fixed Point Theorems for Generalized Contractions and Their Applications
}

\author{
Muhammad Shoaib and Muhammad Sarwar \\ Department of Mathematics, University of Malakand, Chakdara, Dir (Lower), Khyber Pakhtunkhwa 18800, Pakistan \\ Correspondence should be addressed to Muhammad Sarwar; sarwarswati@gmail.com
}

Received 31 July 2016; Accepted 14 September 2016

Academic Editor: Nan-Jing Huang

Copyright (C) 2016 M. Shoaib and M. Sarwar. This is an open access article distributed under the Creative Commons Attribution License, which permits unrestricted use, distribution, and reproduction in any medium, provided the original work is properly cited.

We give common hybrid fixed point results for generalized $(\psi, \phi)$ weak contraction satisfying owc and CLR properties in the framework of metric spaces. An application to functional equations is also discussed.

\section{Introduction and Preliminaries}

Metric fixed point theory provides one of the best important and useful techniques for the existence of fixed point, coupled fixed point, coincidence point, and common fixed point for self-map under different situations. It is applicable for the solution of fractional differential equations, matrix equations, integral equations, functional equations, linear inequalities, or integrodifferential equations (see $[1,2])$. In this area Banach [3] has sorted out fruitful and well known result; such result was later on called Banach contraction principle.

Banach contraction principle generalizes in numerous spaces [4, 5]; mainly, in 1969, Nadler [6] further modified and elaborated the Banach contraction principle to set-valued mapping with the Hausdorff metric. He proved the following theorem.

Theorem 1 (see [6]). Map $f: \widetilde{N} \rightarrow \mathrm{CB}(\widetilde{N})$ on complete $(\widetilde{N}, d)$ metric space which holds the condition

$$
H(f \xi, f \zeta) \leq k d(\xi, \zeta) \quad \forall \xi, \zeta \in \widetilde{N} .
$$

Here, $k \in(0,1), \mathrm{CB}(\widetilde{N})$ are the collection of bounded closed and nonempty subset of $\widetilde{N}$. Then $f$ has a fixed point.

By this virtue fixed point theory has been usefully applicable to various disciplines to solve integral inclusion and functional equations and so forth.

Kutbi and Sintunavarat [7] investigated fixed point theorems for generalized $w_{\alpha}$-contraction multivalued mappings in $\alpha$-complete metric spaces. Shen and Hong [8] demonstrated common fixed point results using generalized contractive multivalued operators in complete metric space.

In Hilbert space, Alber and Guerre-Delabriere [4] presented weak contraction by generalizing contraction further and showed the presence of fixed points for a self-map. Rhoades [9] proved this results in metric space under $\phi$ weak contraction. Dutta and Choudhury [10] generalized $\phi$ weak contraction to the concept of $(\psi, \phi)$ weak contraction and examined results for fixed point. In [11] new fixed point results for $(\alpha, \psi, \xi)$-contractive multivalued mappings on $\alpha$ complete metric spaces and their consequences are studied. Zhang and Song [12] described weak contraction under two self-maps. The result proved by Zhang and Song [12] generalized by Đorić [13] for the presence of common fixed point under $(\psi, \phi)$ weak contraction.

Jungck [14] originated the clue of commutativity of mappings. The idea of commutativity of mappings generalized by the same researcher to compatible mappings [15], later to weakly compatible mappings [16]. Lately, Al-Thagafi and Shahzad considered the notion of occasionally weakly compatible (owc) self-mappings [17] which generalized nontrivial weakly compatible self-maps.

Theory of hybrid fixed point (single-valued and multivalued maps) is a new region in the field of multivalued contractive theory; see [18-20]. Particularly, Aliouche and Popa [21] demonstrated some results for two maps for occasionally weakly compatible hybrid mappings in symmetric space and discuss their application. 
In 1989, Kaneko and Sessa [22] extended the notion of compatible mapping to hybrid mapping. Subsequently Jungck [15] presented the notion of weakly compatible for hybrid (single- and multivalued mapping). In 2007, Abbas and Rhoades [23] defined owc property for hybrid mapping.

The concept of (E.A)-property was introduced by Aamri and El Moutawakil [24]. Sintunavarat and Kumam [25] proved that for the existence of common fixed point, (E.A)property always required the completeness of the subspace and gave the idea of common limit in the range property (CLR) to overcome the mention drawback. Abdou [26] generalized this property to one pair of hybrid (multivalued) maps and then the same author in [27] extended the CLR property to two pairs of hybrid mapping. In [28] common fixed point theorems in fuzzy metric spaces with CLRg property are studied.

In the current work we derived some common hybrid fixed point results for $(\psi, \phi)$ weak contraction in metric space. Throughout the paper $\mathbb{R}^{+}, \mathbb{N}$, and $\mathbb{N}_{0}$ stand for the set of all nonnegative real numbers, the set of positive integers, and the set of nonnegative integers, respectively.

Definition 2. Suppose $\widetilde{N}$ is nonempty set and let $d: \widetilde{N} \times \widetilde{N} \rightarrow$ $\mathbb{R}^{+}$be a function satisfying the following conditions:

(1) $d(\xi, \zeta)=0$ implies that $\xi=\zeta \forall \xi, \zeta \in \widetilde{N}$.

(2) $d(\xi, \zeta)>0 \forall \xi \neq \zeta, \xi, \zeta \in \widetilde{N}$.

(3) $d(\xi, \zeta)=d(\zeta, \xi)$, where $\xi, \zeta \in \widetilde{N}$.

(4) $d(\xi, \zeta) \leq d(\xi, \widetilde{z})+d(\zeta, \widetilde{z}) \forall \xi, \zeta, \widetilde{z} \in \widetilde{N}$.

Then $d$ is a metric on $\widetilde{N}$ and the pair $(\widetilde{N}, d)$ is called metric space.

Definition 3 (see [23]). Maps $f: \widetilde{N} \rightarrow \widetilde{N}, S: \widetilde{N} \rightarrow \mathrm{CB}(\widetilde{N})$ are said to be occasionally weakly compatible (shortly, (owc)property) if $f S \xi \subset S f \xi$ for some $\xi \in \widetilde{N}$ with $f \xi \in S \xi$.

Lemma 4 (see [29]). Let $\widehat{E}$ be a set and $R, S: \widehat{E} \rightarrow \mathbb{R}$ be mappings. If $\mathrm{opt}_{\zeta \in \widehat{E}} R(\zeta)$ and $\mathrm{opt}_{\zeta \in \widehat{E}} S(\zeta)$ are bounded, then

$$
\left|\mathrm{opt}_{\zeta \in \widehat{E}} R(\zeta)-\mathrm{opt}_{\zeta \in \widehat{E}} S(\zeta)\right| \leq \sup _{\zeta \in \widehat{E}}|R(\zeta)-S(\zeta)| .
$$

Definition 5 (see [15]). Maps $f: \widetilde{N} \rightarrow \widetilde{N}, S: \widetilde{N} \rightarrow \mathrm{CB}(\widetilde{N})$ on metric space $(\widetilde{N}, d)$ are said to be weakly compatible if $f S \xi=$ $S f \xi$ whenever $f \xi \in S \xi$.

Definition 6 (see [25]). Maps $f, g: \widetilde{N} \rightarrow \widetilde{N}$ are said to satisfy the common limit in the range of $f$ with respect to $g$ (shortly, the $\left(\mathrm{CLR}_{f}\right)$-property with respect to $\left.g\right)$ if there exists a sequence $\xi_{n}$ in $\widetilde{N}$ such that, for some $u \in \widetilde{N}, \lim _{n \rightarrow \infty} f \xi_{n}=$ $\lim _{n \rightarrow \infty} g \xi_{n}=f u$.

The following definitions can be found in [27].

Definition 7. Mappings $f: \widetilde{N} \rightarrow \widetilde{N}, S: \widetilde{N} \rightarrow \mathrm{CB}(\widetilde{N})$ on $(\widetilde{N}, d)$ metric space are said to satisfy the common limit in the range of $f$ with respect to $S$ (shortly, the $\left(\mathrm{CLR}_{f}\right)$-property with respect to $S$ ) if there exists a sequence $x_{n}$ in $\widetilde{N}$ and $\Omega_{1} \epsilon$ $\mathrm{CB}(\widetilde{N})$ such that, for some $u \in \widetilde{N}, \lim _{n \rightarrow \infty} f \xi_{n}=f u \in \Omega_{1}=$ $\lim _{n \rightarrow \infty} S \xi_{n}=f u$.

Definition 8. Mappings $f, g: \widetilde{N} \rightarrow \widetilde{N}$ and $S, T: \widetilde{N} \rightarrow \mathrm{CB}(\widetilde{N})$ on metric space $(\widetilde{N}, d)$ are said to satisfy the common limit in the range of $f$ with respect to $S$ (shortly, the $\left(\mathrm{CLR}_{f}\right)$-property with respect to $S$ ) if there exist sequences $\xi_{n}$ and $\zeta_{n}$ in $\widetilde{N}$ and $\Omega_{1}, \Omega_{2} \in \operatorname{CB}(\widetilde{N})$ such that, for some $u \in \widetilde{N}, \lim _{n \rightarrow \infty} S \xi_{n}=$ $\Omega_{1}, \lim _{n \rightarrow \infty} T \zeta_{n}=\Omega_{2}$, and $\lim _{n \rightarrow \infty} f \xi_{n}=\lim _{n \rightarrow \infty} g \zeta_{n}=f u \epsilon$ $\Omega_{1} \cap \Omega_{2}$.

Now, we give some definitions for multivalued mappings defined in a metric space $(\widetilde{N}, d)$. Define the function $H$ : $\mathrm{CB}(\widetilde{N}) \times \mathrm{CB}(\widetilde{N}) \rightarrow \mathbb{R}^{+}$for $\Omega_{1}, \Omega_{2} \in \mathrm{CB}(\widetilde{N})$ by

$$
H\left(\Omega_{1}, \Omega_{2}\right)=\max \left\{\sup _{\varsigma \in \Omega_{1}} d\left(\varsigma, \Omega_{2}\right), \sup _{\zeta \in \Omega_{1}} d\left(\zeta, \Omega_{1}\right)\right\},
$$

where

$$
d\left(\xi, \Omega_{1}\right)=\inf \left\{d(\xi, \zeta): \zeta \in \Omega_{1}\right\}
$$

where

$$
\begin{aligned}
& \delta\left(\Omega_{1}, \Omega_{2}\right)=\sup \left\{d(\varsigma, \zeta): \varsigma \in \Omega_{1}, \zeta \in \Omega_{2}\right\}, \\
& D\left(\Omega_{1}, \Omega_{2}\right)=\inf \left\{d(\varsigma, \zeta): \varsigma \in \Omega_{1}, \zeta \in \Omega_{2}\right\} .
\end{aligned}
$$

The following can be deduced from the definition of $\delta$ :

$$
\begin{aligned}
& \delta\left(\Omega_{1}, \Omega_{2}\right)=\delta\left(\Omega_{2}, \Omega_{1}\right) \\
& \delta\left(\Omega_{1}, \Omega_{3}\right) \leq \delta\left(\Omega_{1}, \Omega_{2}\right)+\delta\left(\Omega_{2}, \Omega_{3}\right), \\
& \delta\left(\Omega_{1}, \Omega_{2}\right)=0 \Longleftrightarrow \Omega_{1}=\Omega_{2}=\{\varsigma\}, \\
& \delta\left(\Omega_{1}, \Omega_{1}\right)=\operatorname{diam} \Omega_{1} .
\end{aligned}
$$

Lemma 9. Let $(\widetilde{N}, d)$ be a metric space. For any $\Omega_{1}, \Omega_{2}, \Omega_{3} \in$ $\mathrm{CB}(\widetilde{N})$ and $\xi, \zeta \in \widetilde{N}$, we have the following:

(1) $d\left(\xi, \Omega_{2}\right) \leq d(\xi, \zeta), \forall \zeta \in \Omega_{2}$.

(2) $\delta\left(\Omega_{1}, \Omega_{2}\right) \leq H\left(\Omega_{1}, \Omega_{2}\right)$.

(3) $d\left(\xi, \Omega_{2}\right) \leq H\left(\Omega_{1}, \Omega_{2}\right), \forall \xi \in \Omega_{1}$.

(4) $H\left(\Omega_{1}, \Omega_{1}\right)=0$.

(5) $H\left(\Omega_{1}, \Omega_{2}\right)=H\left(\Omega_{2}, \Omega_{1}\right)$.

(6) $H\left(\Omega_{1}, \Omega_{3}\right) \leq H\left(\Omega_{1}, \Omega_{2}\right)+H\left(\Omega_{2}, \Omega_{3}\right)$.

(7) $H\left(\xi, \Omega_{1}\right) \leq d(\xi, \zeta)+d\left(\zeta, \Omega_{1}\right)$.

Lemma 10 (see [6]). Assume a metric space $(\widetilde{N}, d)$. Furthermore, $\Omega_{1}, \Omega_{2} \in \mathrm{CB}(\widetilde{N})$; then for every $h>1$ and for each $\varsigma \in \Omega_{1}$ there exist $\zeta(\varsigma) \in \Omega_{2}$ such that $d(\varsigma, \zeta) \leq h H\left(\Omega_{1}, \Omega_{2}\right)$. $h \geq 1$.

In [6] it is shown that the above lemma is also true for

Lemma 11. Assume a metric space $(\widetilde{N}, d)$. Furthermore, $\Omega_{1}$, $\Omega_{2} \in \mathrm{CB}(\widetilde{N})$; then for every $h \geq 1$ and for each $\varsigma \in \Omega_{1}$ there exist $\zeta(\varsigma) \in \Omega_{2}$ such that $d(\varsigma, \zeta) \leq h H\left(\Omega_{1}, \Omega_{2}\right)$. 


\section{Main Results}

In our main results we used the following two classes:

$$
\begin{aligned}
& \Psi=\left\{\psi: \mathbb{R}^{+} \longrightarrow \mathbb{R}^{+} \text {is continuous and nondecreasing function such that } \psi(\tau)=0 \text { iff } \tau=0\right\} . \\
& \Phi=\left\{\phi: \mathbb{R}^{+} \longrightarrow \mathbb{R}^{+} \text {is a lower semicontinuous and nondecreasing function such that } \phi(\tau)=0 \text { iff } \tau=0\right\} .
\end{aligned}
$$

Theorem 12. Let $f, g: \widetilde{N} \rightarrow \widetilde{N}, S, T: \widetilde{N} \rightarrow B(\widetilde{N})$ on metric space $(\widetilde{N}, d)$. Furthermore assume that $(S, g)$ and $(T, f)$ have (owc)-property and

$$
\psi\left(\delta^{p}\left(T \zeta, S_{\varsigma}\right)\right) \leq \psi(\Theta(\zeta, \varsigma))-\phi(\Theta(\zeta, \varsigma)),
$$

where

$$
\begin{aligned}
& \Theta(\zeta, \varsigma)=\max \left\{d^{p}(f \zeta, g \varsigma), \frac{d^{p}(f \zeta, T \zeta) d^{p}(g \varsigma, S \varsigma)}{1+d^{p}(f \zeta, g \varsigma)},\right. \\
& \left.\frac{d^{p}(f \zeta, S \varsigma) d^{p}(g \varsigma, T \zeta)}{1+d^{p}(f \zeta, g \varsigma)}\right\} .
\end{aligned}
$$

Here, $\psi \in \Psi, \phi \in \Phi$ and $p \geq 1$, there exists unique common fixed point of $f, g, S$, and $T$.

Proof. Since, $(S, g),(T, f)$ pairs hold (owc)-property, therefore, there exist $v, u \in \widetilde{N}$ such that

$$
\begin{gathered}
g v \in S v, \\
g S v \subset S g v, \\
f u \in T u, \\
f T u \subset T f u,
\end{gathered}
$$

which implies that $g g v \in S g v$ and $f f u \in T f u$.

Now, we prove that $g v=f u$. Suppose $g v \neq f u$; then, from (8), we have

$$
\psi\left(\delta^{p}(T u, S v)\right) \leq \psi(\Theta(u, v))-\phi(\Theta(u, v)),
$$

where

$$
\begin{aligned}
& \Theta(u, v)=\max \left\{d^{p}(f u, g v), \frac{d^{p}(f u, T u) d^{p}(g v, S v)}{1+d^{p}(f u, g v)},\right. \\
& \left.\frac{d^{p}(f u, S v) d^{p}(g v, T u)}{1+d^{p}(f u, g v)}\right\},
\end{aligned}
$$

and we have

$$
\begin{aligned}
& \frac{d^{p}(f u, S v) d^{p}(g v, T u)}{1+d^{p}(f u, g v)} \leq \frac{d^{p}(f u, g v) d^{p}(g v, f u)}{1+d^{p}(f u, g v)} \\
& <d^{p}(f u, g v) .
\end{aligned}
$$

If

$$
\Theta(u, v)=d^{p}(f u, g v)
$$

then, (11) becomes

$$
\begin{aligned}
\psi\left(\delta^{p}(T u, S v)\right) & \leq \psi\left(d^{p}(f u, g v)\right)-\phi\left(d^{p}(f u, g v)\right) \\
& <\psi\left(d^{p}(f u, g v)\right) .
\end{aligned}
$$

But $\psi$ is nondecreasing, so $\delta^{p}(T u, S v)<d^{p}(f u, g v)$. Since $f u \in T u$ and $g v \in S v$, therefore, we have

$$
d^{p}(f u, g v) \leq \delta^{p}(T u, S v)<d^{p}(f u, g v),
$$

which gives us contradiction. Hence $f u=g v$.

If

$$
\Theta(u, v)=\frac{d^{p}(f u, S v) d^{p}(g v, T u)}{1+d^{p}(f u, g v)}
$$

then, (11) becomes

$$
\begin{aligned}
\psi\left(\delta^{p}(T u, S v)\right) \leq & \psi\left(\frac{d^{p}(f u, S v) d^{p}(g v, T u)}{1+d^{p}(f u, g v)}\right) \\
& -\phi\left(\frac{d^{p}(f u, S v) d^{p}(g v, T u)}{1+d^{p}(f u, g v)}\right) \\
\leq & \psi\left(d^{p}(f u, g v)\right) \\
& -\phi\left(\frac{d^{p}(f u, S v) d^{p}(g v, T u)}{1+d^{p}(f u, g v)}\right) \\
< & \psi\left(d^{p}(f u, g v)\right)
\end{aligned}
$$

Since, $\psi$ is nondecreasing and $f u \in T u$ and $g v \in S v$, therefore, one can get

$$
d^{p}(f u, g v) \leq \delta^{p}(T u, S v)<d^{p}(f u, g v),
$$

which is again not possible. Hence $f u=g v$. Similarly it is easy to show in the case if

$$
\Theta(u, v)=\frac{d^{p}(f u, T u) d^{p}(g v, S v)}{1+d^{p}(f u, g v)},
$$

then $g v=f u$.

Next, we have to show that $f u$ is a fixed point of $f$. Assume that $f f u \neq f u$. Then, by using (8), we have

$$
\begin{aligned}
\psi\left(d^{p}(f f u, f u)\right) & =\psi\left(d^{p}(f f u, g v)\right) \\
& \leq \psi\left(\delta^{p}(T f u, S v)\right) \\
& \leq \psi(\Theta(f u, v))-\phi(\Theta(f u, v)) .
\end{aligned}
$$


Here,

$$
\begin{aligned}
& \Theta(f u, v)=\max \left\{d^{p}(f f u, g v),\right. \\
& \frac{d^{p}(f f u, S u) d^{p}(g v, T v)}{1+d^{p}(f f u, g v)}, \\
& \left.\frac{d^{p}(f f u, T v) d^{p}(g v, S f u)}{1+d^{p}(f f u, g v)}\right\}, \\
& \frac{d^{p}(f f u, T v) d^{p}(g v, S f u)}{1+d^{p}(f S u, g v)} \leq d^{p}(f f u, T v)<d^{p}(f f u, \\
& g v) .
\end{aligned}
$$

If

$$
\Theta(f u, v)=d^{p}(f f u, g v),
$$

then, (21) implies that

$$
\begin{aligned}
\psi\left(d^{p}(f f u, f u)\right)= & \psi\left(d^{p}(f f u, g v)\right) \\
\leq & \psi\left(\delta^{p}(T f u, S v)\right) \\
\leq & \psi\left(d^{p}(f f u, g v)\right) \\
& -\phi\left(d^{p}(f f u, g v)\right) \\
< & \psi\left(d^{p}(f f u, g v)\right) .
\end{aligned}
$$

If

$$
\Theta(f u, v)=\frac{d^{p}(f f u, T v) d^{p}(g v, S f u)}{1+d^{p}(f f u, g v)},
$$

then, (21) implies that

$$
\begin{aligned}
\psi\left(d^{p}(f f u, f u)\right) & \\
\leq & \psi\left(\frac{d^{p}(f f u, T v) d^{p}(g v, S f u)}{1+d^{p}(f f u, g v)}\right) \\
& -\phi\left(\frac{d^{p}(f f u, T v) d^{p}(g v, S f u)}{1+d^{p}(f f u, g v)}\right) \\
\leq & \left(d^{p}(f f u, g v)\right) \\
& -\phi\left(\frac{d^{p}(f f u, T v) d^{p}(g v, S f u)}{1+d^{p}(f f u, g v)}\right) \\
< & \psi\left(d^{p}(f f u, g v)\right) .
\end{aligned}
$$

Similarly it gives us contradiction if

$$
\Theta(f u, v)=\frac{d^{p}(f f u, S u) d^{p}(g v, T v)}{1+d^{p}(f f u, g v)},
$$

which gives us contradiction in all three cases. Hence $f f u=$ $f u$. Similarly, we can prove $f u=g f u=f f u$. Thus we have

$$
\begin{aligned}
& f u=f f u \in T f u, \\
& f u=g f u \in S g u=S f u .
\end{aligned}
$$

Therefore, $f u$ is a common fixed point of $f, g, T$, and $S$. Furthermore, by using condition (8), we have

$$
\begin{aligned}
\psi\left(\delta^{p}(T f u, S f u)\right) \leq & \psi(\Theta(f u, f u)) \\
& -\phi(\Theta(f u, f u)),
\end{aligned}
$$

where

$$
\begin{aligned}
& \Theta(f u, f u)=\max \left\{d^{p}(f f u, g f u),\right. \\
& \frac{d^{p}(f f u, S f u) d^{p}(g f u, T f u)}{1+d^{p}(f f u, g f u)}, \\
& \left.\frac{d^{p}(f f u, T f u) d^{p}(g f u, S f u)}{1+d^{p}(f f u, g f u)}\right\}=\max \{0,0,0\},
\end{aligned}
$$

which implies that $T f u=S f u=\{f u\}$.

Next, to show that fixed is unique, suppose there exists another fixed $\varsigma_{1}$ other than $\varsigma_{2}$; then, from condition (8), we have

$$
\begin{aligned}
\psi\left(\delta^{p}\left(\varsigma_{2}, \varsigma_{1}\right)\right) & =\psi\left(\delta^{p}\left(T \varsigma_{2}, S \varsigma_{1}\right)\right) \\
& \leq \psi\left(\Theta\left(\varsigma_{2}, \varsigma_{1}\right)\right)-\phi\left(\Theta\left(\varsigma_{2}, \varsigma_{1}\right)\right)
\end{aligned}
$$

where

$$
\begin{gathered}
\Theta\left(\varsigma_{2}, \varsigma_{1}\right)=\max \left\{d^{p}\left(f \varsigma_{2}, g \varsigma_{1}\right),\right. \\
\frac{d^{p}\left(f \varsigma_{2}, S \varsigma_{2}\right) d^{p}\left(g \varsigma_{1}, T \varsigma_{1}\right)}{1+d^{p}\left(f \varsigma_{2}, g \varsigma_{1}\right)}, \\
\left.\frac{d^{p}\left(f \varsigma_{2}, T \varsigma_{1}\right) d^{p}\left(g \varsigma_{1}, S \varsigma_{2}\right)}{1+d^{p}\left(f \varsigma_{2}, g \varsigma_{1}\right)}\right\} .
\end{gathered}
$$

By simple calculation we conclude that the common fixed point is unique.

Theorem 13. Let $f, g: \widetilde{N} \rightarrow \widetilde{N}, S, T: \widetilde{N} \rightarrow B(\widetilde{N})$ on metric space $(\widetilde{N}, d)$. Furthermore, assume $(S, f)$ and $(T, g)$ satisfy the $\left(C L R_{f}\right)$-property and

$$
\psi\left(H^{p}(S \zeta, T \varsigma)\right) \leq \psi(\Theta(\zeta, \varsigma))-\phi(\Theta(\zeta, \varsigma))
$$

where

$$
\begin{aligned}
& \Theta(\zeta, \varsigma)=\max \left\{d^{p}(f \zeta, g \varsigma), \frac{d^{p}(f \zeta, S \zeta) d^{p}(g \varsigma, T \varsigma)}{1+d^{p}(f \zeta, g \varsigma)},\right. \\
& \left.\frac{d^{p}(f \zeta, T \varsigma) d^{p}(g \varsigma, S \zeta)}{1+d^{p}(f \zeta, g \varsigma)}\right\},
\end{aligned}
$$

$p \geq 1, \psi \in \Psi$, and $\phi \in \Phi$. Further assume the following conditions also hold:

(i) $f$, S have a coincidence point.

(ii) $g, T$ have a coincidence point. 
(iii) If $f$, S are weakly compatible at $u$ and, moreover, $f f u=$ $f u \forall u \in C(f, S)$, then, $f$, S have a common fixed point.

(iv) If $g, T$ are weakly compatible at $u$ and, moreover, ggu $=$ $g u \forall u \in C(g, T)$, then, $g$, $T$ have a common fixed point.

(v) If both conditions (iii) and (iv) hold, then, there exist common fixed point of $f, g, S$, and $T$.

Proof. Since $(S, f)$ and $(T, g)$ satisfy the $\left(\mathrm{CLR}_{f}\right)$-property, there exists two sequences $\zeta_{n}, \varsigma_{n} \in \widetilde{N}$ and $A, B \in \mathrm{CB}(\widetilde{N})$ such that

$$
\begin{aligned}
& \lim _{n \rightarrow \infty} S \zeta_{n}=B, \\
& \lim _{n \rightarrow \infty} T \zeta_{n}=A, \\
& \lim _{n \rightarrow \infty} f \zeta_{n}=\lim _{n \rightarrow \infty} g \zeta_{n}=f u \in A \cap B \quad \text { for some } u \in \widetilde{N}
\end{aligned}
$$

and, by closeness of $f \widetilde{N}$ and $g \widetilde{N}$, we have

$$
\begin{aligned}
& f u=g v, \\
& f u=g w
\end{aligned}
$$

for some $v, w \in \widetilde{N}$.

Now, we claim that $g w \in T w$. To Justify the said claim, using condition (33) with $\zeta=\zeta_{n}$ and $\varsigma=w$, we have

$$
\psi\left(H^{p}\left(S \zeta_{n}, T w\right)\right) \leq \psi\left(\Theta\left(\zeta_{n}, w\right)\right)-\phi\left(\Theta\left(\zeta_{n}, w\right)\right),
$$

where

$$
\begin{gathered}
\Theta\left(\zeta_{n}, w\right)=\max \left\{d^{p}\left(f \zeta_{n}, g w\right),\right. \\
\frac{d^{p}\left(f \zeta_{n}, S \zeta_{n}\right) d^{p}(g w, T w)}{1+d^{p}\left(f \zeta_{n}, g w\right)}, \\
\left.\frac{d^{p}\left(f \zeta_{n}, T w\right) d^{p}\left(g w, S \zeta_{n}\right)}{1+d^{p}\left(f \zeta_{n}, g w\right)}\right\} .
\end{gathered}
$$

By taking limit, we get

$$
\begin{aligned}
\psi\left(H^{p}(B, T w)\right) \leq & \lim _{n \rightarrow \infty} \psi\left(\Theta\left(\zeta_{n}, w\right)\right) \\
& -\lim _{n \rightarrow \infty} \phi\left(\Theta\left(\zeta_{n}, w\right)\right),
\end{aligned}
$$

where

$$
\begin{aligned}
& \lim _{n \rightarrow \infty} \Theta\left(\zeta_{n}, w\right)=\lim _{n \rightarrow \infty} \max \left\{d^{p}(f v, g w),\right. \\
& \left.\quad \frac{d^{p}(f v, B) d^{p}(g w, T w)}{1+d^{p}(f v, g w)}, \frac{d^{p}(f v, T w) d^{p}(g w, B)}{1+d^{p}(f v, g w)}\right\} \\
& =\max \{0,0,0\} .
\end{aligned}
$$

Equation (39) becomes

$$
\psi\left(H^{p}(B, T w)\right)=0,
$$

which implies that

$$
H^{p}(B, T w)=0 .
$$

Since, $g w \in B$, by definition of Hausdorff metric

$$
d^{p}(g w, T w) \leq H(B, T w)=0 .
$$

Hence we have $g w \in T w$.

In the other side using condition (33) with $\zeta=v$ and $\varsigma=$ $\varsigma_{n}$, we have

$$
\psi\left(H^{p}\left(S v, T \varsigma_{n}\right)\right) \leq \psi\left(\Theta\left(v, \varsigma_{n}\right)\right)-\phi\left(\Theta\left(v, \varsigma_{n}\right)\right) ;
$$

here,

$$
\begin{gathered}
\Theta\left(v, \varsigma_{n}\right)=\max \left\{d\left(f v, g \varsigma_{n}\right),\right. \\
\frac{d^{p}(f v, S v) d^{p}\left(g \varsigma_{n}, T \varsigma_{n}\right)}{1+d^{p}\left(f v, g \varsigma_{n}\right)}, \\
\left.\frac{d^{p}\left(f v, T \varsigma_{n}\right) d^{p}\left(g \varsigma_{n}, S v\right)}{1+d^{p}\left(f v, g \varsigma_{n}\right)}\right\} .
\end{gathered}
$$

By taking limit, we get

$$
\begin{aligned}
\psi\left(H^{p}(S v, A)\right) \leq & \psi\left(\lim _{n \rightarrow \infty} \Theta\left(v, \varsigma_{n}\right)\right) \\
& -\lim _{n \rightarrow \infty} \phi\left(\Theta\left(v, \varsigma_{n}\right)\right),
\end{aligned}
$$

where

$$
\begin{aligned}
& \lim _{n \rightarrow \infty} \Theta\left(v, \varsigma_{n}\right)=\lim _{n \rightarrow \infty} \max \left\{d^{p}(f v, g w),\right. \\
& \left.\quad \frac{d^{p}(f v, S v) d^{p}(g w, A)}{1+d^{p}(f v, g w)}, \frac{d^{p}(f v, A) d^{p}(g w, S v)}{1+d^{p}(f v, g w)}\right\} \\
& =\max \{0,0,0\}
\end{aligned}
$$

condition (33) becomes

$$
\begin{aligned}
\psi\left(H^{p}(S v, A)\right) \leq & \psi\left(\lim _{n \rightarrow \infty} \Theta\left(\zeta, \varsigma_{n}\right)\right) \\
& -\lim _{n \rightarrow \infty} \phi\left(\Theta\left(\zeta, \varsigma_{n}\right)\right) ;
\end{aligned}
$$

which implies

$$
\psi\left(H^{p}(S v, A)\right)=0
$$

from this, we have

$$
H^{p}(S v, A)=0 .
$$

By definition of Hausdorff metric

$$
d^{p}(f v, S v) \leq H^{p}(A, S v)=0 \quad \text { therfore } f v \in S v .
$$

Thus, maps $g, T$ have a coincidence point $w$ and the maps $f, S$ have a coincidence point $v$. Furthermore, by virtue of condition (ii) we obtain $f f u=f u$ and $f f u \in S f u$. So, $u=f u \in S u$; this proved (iii). A similar argument proves (iv); that is, $u=g u \in T u$. From $u=f u \in S u, u=g u \in T u$, we deduced (v). 


\section{Applications to System of Functional Equations}

In this section, we discuss common solution for four functional equations given below with the help of Theorems 12 and 13. Throughout this unit opt signifies inf or sup, $\widehat{Z}$ and $\widehat{Y}$ stand for Banach spaces, the state space is $\widetilde{E} \subset \widehat{Z}$, the decision space is $\widetilde{F} \subset \widehat{Y}$, and the space of all bounded real-valued functions on $\widetilde{E}$ is $X=B(\widetilde{E})$ which is Banach space. Define $d: X \times X \rightarrow \mathbb{R}^{+}$, by

$$
d\left(\dot{u}_{1}, \dot{u}_{2}\right)=\sup _{x \in \widetilde{E}}\left|\dot{u}_{1}(x)-\dot{u}_{2}(x)\right|=\left\|\dot{u}_{1}-\dot{u}_{2}\right\| .
$$

Here,

$$
\|\dot{u}\|=\sup \left\{|\dot{u}(x)|: \xi_{11}^{\star} \in \widetilde{E}\right\} \quad \forall \dot{u} \in B(\widetilde{E})
$$

and $\mu, v: \widetilde{E} \times \widetilde{F} \rightarrow \mathbb{R}, b_{i}: \widetilde{E} \times \widetilde{F} \rightarrow \widetilde{E}, \Psi_{i}: \widetilde{E} \times \widetilde{F} \times \mathbb{R} \rightarrow \mathbb{R}$

Consider the system

$$
\begin{array}{ll}
g_{1}\left(\xi_{11}^{\star}\right)=\text { opt }_{\xi_{22}^{\star} \in \widetilde{F}}\left\{\mu\left(\xi_{11}^{\star}, \xi_{22}^{\star}\right)\right. & \\
\left.\quad+\Psi_{1}\left(\xi_{11}^{\star}, \xi_{22}^{\star}, g_{1}\left(b_{1}\left(\xi_{11}^{\star}, \xi_{22}^{\star}\right)\right)\right)\right\} \quad \forall \xi_{11}^{\star} \in \widetilde{E}, \\
g_{2}\left(\xi_{11}^{\star}\right)=\operatorname{opt}_{\xi_{22}^{\star} \in \widetilde{F}}\left\{\mu\left(\xi_{11}^{\star}, \xi_{22}^{\star}\right)\right. & \\
\left.\quad+\Psi_{2}\left(\xi_{11}^{\star}, \xi_{22}^{\star}, g_{2}\left(b_{2}\left(\xi_{11}^{\star}, \xi_{22}^{\star}\right)\right)\right)\right\} \quad \forall \xi_{11}^{\star} \in \widetilde{E}, \\
g_{3}\left(\xi_{11}^{\star}\right)=\text { opt }_{\xi_{22}^{\star} \in \widetilde{F}}\left\{\nu\left(\xi_{11}^{\star}, \xi_{22}^{\star}\right)\right. \\
\left.\quad+\Psi_{3}\left(\xi_{11}^{\star}, \xi_{22}^{\star}, g_{3}\left(b_{3}\left(\xi_{11}^{\star}, \xi_{22}^{\star}\right)\right)\right)\right\} \quad \forall \xi_{11}^{\star} \in \widetilde{E}, \\
g_{4}\left(\xi_{11}^{\star}\right)=\text { opt }_{\xi_{22}^{\star} \in \widetilde{F}}\left\{v\left(\xi_{11}^{\star}, \xi_{22}^{\star}\right)\right. & \\
\left.\quad+\Psi_{4}\left(\xi_{11}^{\star}, \xi_{22}^{\star}, g_{4}\left(b_{4}\left(\xi_{11}^{\star}, \xi_{22}^{\star}\right)\right)\right)\right\} \quad \forall \xi_{11}^{\star} \in \widetilde{E} .
\end{array}
$$

Here $\xi_{11}^{\star}$ and $\xi_{22}^{\star}$ denote the state vectors and decision vectors, respectively, $b_{1}, b_{2}, b_{3}$, and $b_{4}$ signify the transformations of the process, and $g_{1}\left(\xi_{11}^{\star}\right), g_{2}\left(\xi_{11}^{\star}\right), g_{3}\left(\xi_{11}^{\star}\right)$, and $g_{4}\left(\xi_{11}^{\star}\right)$ symbolized the optimal return functions under the initial state $\xi_{11}^{\star}$.

Let $S, T, f, g: B(\widetilde{E}) \rightarrow B(\widetilde{E})$ be defined by

$$
\begin{aligned}
& \operatorname{Sh}\left(\xi_{11}^{\star}\right)=\mathrm{opt}_{\xi_{22}^{\star} \in \widetilde{F}}\left\{\mu\left(\xi_{11}^{\star}, \xi_{22}^{\star}\right)\right. \\
& \left.+\Psi_{1}\left(\xi_{11}^{\star}, \xi_{22}^{\star}, h\left(b_{1}\left(\xi_{11}^{\star}, \xi_{22}^{\star}\right)\right)\right)\right\}, \\
& \operatorname{Th}\left(\xi_{11}^{\star}\right)=\mathrm{opt}_{\xi_{22}^{\star} \in \widetilde{F}}\left\{\mu\left(\xi_{11}^{\star}, \xi_{22}^{\star}\right)\right. \\
& \left.+\Psi_{2}\left(\xi_{11}^{\star}, \xi_{22}^{\star}, h\left(b_{2}\left(\xi_{11}^{\star}, \xi_{22}^{\star}\right)\right)\right)\right\}, \\
& f h\left(\xi_{11}^{\star}\right)=\text { opt }_{\xi_{22} \in \widetilde{F}}\left\{\nu\left(\xi_{11}^{\star}, \xi_{22}^{\star}\right)\right. \\
& \left.+\Psi_{3}\left(\xi_{11}^{\star}, \xi_{22}^{\star}, h\left(b_{3}\left(\xi_{11}^{\star}, \xi_{22}^{\star}\right)\right)\right)\right\}, \\
& g h\left(\xi_{11}^{\star}\right)=\mathrm{opt}_{\xi_{22}^{\star} \in \widetilde{F}}\left\{\nu\left(\xi_{11}^{\star}, \xi_{22}^{\star}\right)\right. \\
& \left.\quad+\Psi_{4}\left(\xi_{11}^{\star}, \xi_{22}^{\star}, h\left(b_{4}\left(\xi_{11}^{\star}, \xi_{22}^{\star}\right)\right)\right)\right\},
\end{aligned}
$$

where $\left(\xi_{11}^{\star}, h\right) \in \widetilde{E} \times B(\widetilde{E})$.
Theorem 14. Assume $S, T, f, g: B(\widetilde{E}) \rightarrow B(\widetilde{E})$ are maps given by (55) which hold the following conditions:

(1) $\mu, v$, and $\Psi_{i}$, for $i=1,2,3,4$, are bounded.

(2) $(S, f)$ and $(T, g)$ pairs share (CLR) property.

(3) $(S, f)$ and $(T, g)$ pairs are weakly compatible; further, $f f h=f h$ and $g g h=g h$.

(4) $\forall\left(\xi_{11}^{\star}, y, h, w\right) \in \widetilde{E} \times \widetilde{F} \times B(\widetilde{E}) \times B(\widetilde{E})$,

$$
\begin{aligned}
\mid & \Psi_{1}\left(\xi_{11}^{\star}, \xi_{22}^{\star}, h\left(a_{1}\left(\xi_{11}^{\star}, \xi_{22}^{\star}\right)\right)\right) \\
& -\Psi_{2}\left(\xi_{11}^{\star}, \xi_{22}^{\star}, w\left(a_{2}\left(\xi_{11}^{\star}, \xi_{22}^{\star}\right)\right)\right) \mid \leq \Theta(h, w) ;
\end{aligned}
$$

here

$$
\begin{gathered}
\Theta(h, w)=\max \left\{d^{p}(f h, g w),\right. \\
\frac{d^{p}(f h, S w) d^{p}(g w, T w)}{1+d^{p}(f h, g w)}, \\
\left.\frac{d^{p}(f h, T w) d^{p}(g w, S h)}{1+d^{p}(f h, g w)}\right\} .
\end{gathered}
$$

Then, (54) system equations have a common solution in $B(\widetilde{E})$.

Proof. Since $\mu, \nu$, and $\Psi_{i}$, for $i=1,2,3,4$, are bounded, there exists $M>0$ such that

$$
\begin{gathered}
\sup \left\{\left\|\mu\left(\xi_{11}^{\star}, \xi_{22}^{\star}\right)\right\|,\left\|\nu\left(\xi_{11}^{\star}, \xi_{22}^{\star}\right)\right\|,\left\|\Psi_{i}\left(\xi_{11}^{\star}, \xi_{22}^{\star}, t\right)\right\|:\right. \\
\left.\left(\xi_{11}^{\star}, \xi_{22}^{\star}, t\right) \in \widetilde{E} \times \widetilde{F} \times \mathbb{R}\right\} \leq M .
\end{gathered}
$$

Thus by Lemma 4, (55), and (58) $S, T, f$, and $g$ are selfmappings in $B(\widetilde{E})$.

Let $\left(\xi_{11}^{\star}, h, w\right) \in \widetilde{E} \times B(\widetilde{E}) \times B(\widetilde{E})$. Suppose that opt $\xi_{22}^{\star} \in \widetilde{F}=$ $\inf _{\xi_{22}^{\star} \in \widetilde{F}}$. Then using (55) we can find $\xi_{22}^{\star}, \xi_{33}^{\star} \in \widetilde{F}$ such that

$$
\begin{aligned}
\operatorname{Sh}\left(\xi_{11}^{\star}\right)> & \mu\left(\xi_{11}^{\star}, \xi_{22}^{\star}\right)+\Psi_{1}\left(\xi_{11}^{\star}, \xi_{22}^{\star}, h\left(b_{1}\left(\xi_{11}^{\star}, \xi_{22}^{\star}\right)\right)\right) \\
& -\delta ; \\
T w\left(\xi_{11}^{\star}\right)> & \mu\left(\xi_{11}^{\star}, \xi_{33}^{\star}\right) \\
& +\Psi_{2}\left(\xi_{11}^{\star}, \xi_{33}^{\star}, w\left(b_{2}\left(\xi_{11}^{\star}, \xi_{33}^{\star}\right)\right)\right)-\delta ; \\
\operatorname{Sh}\left(\xi_{11}^{\star}\right) \leq \mu & \mu\left(\xi_{11}^{\star}, \xi_{33}^{\star}\right) \\
& +\Psi_{1}\left(\xi_{11}^{\star}, \xi_{33}^{\star}, h\left(b_{1}\left(\xi_{11}^{\star}, \xi_{33}^{\star}\right)\right)\right) ; \\
T w\left(\xi_{11}^{\star}\right) \leq \mu & \left(\xi_{11}^{\star}, \xi_{22}^{\star}\right) \\
& +\Psi_{2}\left(\xi_{11}^{\star}, \xi_{22}^{\star}, w\left(b_{2}\left(\xi_{11}^{\star}, \xi_{22}^{\star}\right)\right)\right),
\end{aligned}
$$

where $\left(\xi_{11}^{\star}, h\right) \in \widetilde{E} \times B(\widetilde{E})$. 
Next, with help of (59) and (62), we have

$$
\begin{aligned}
& \operatorname{Sh}\left(\xi_{11}^{\star}\right)-T w\left(\xi_{11}^{\star}\right)>\Psi_{1}\left(\xi_{11}^{\star}, \xi_{22}^{\star}, h\left(b_{1}\left(\xi_{11}^{\star}, \xi_{22}^{\star}\right)\right)\right) \\
& \quad-\Psi_{2}\left(\xi_{11}^{\star}, \xi_{22}^{\star}, w\left(b_{2}\left(\xi_{11}^{\star}, \xi_{22}^{\star}\right)\right)\right)-\delta \\
& \geq-\max \left\{\mid \Psi_{1}\left(\xi_{11}^{\star}, \xi_{22}^{\star}, h\left(b_{1}\left(\xi_{11}^{\star}, \xi_{22}^{\star}\right)\right)\right)\right. \\
& \quad-\Psi_{2}\left(\xi_{11}^{\star}, \xi_{22}^{\star}, w\left(b_{2}\left(\xi_{11}^{\star}, \xi_{22}^{\star}\right)\right)\right) \mid \\
& \mid \Psi_{1}\left(\xi_{11}^{\star}, \xi_{33}^{\star}, h\left(b_{1}\left(\xi_{11}^{\star}, \xi_{33}^{\star}\right)\right)\right) \\
& \left.-\Psi_{2}\left(\xi_{11}^{\star}, \xi_{33}^{\star}, w\left(b_{2}\left(\xi_{11}^{\star}, \xi_{33}^{\star}\right)\right)\right) \mid\right\}-\delta .
\end{aligned}
$$

Similarly, using (60) and (61), we have

$$
\begin{aligned}
& \operatorname{Sh}\left(\xi_{11}^{\star}\right)-T w\left(\xi_{11}^{\star}\right)<\Psi_{1}\left(\xi_{11}^{\star}, \xi_{33}^{\star}, h\left(b_{1}\left(\xi_{11}^{\star}, \xi_{33}^{\star}\right)\right)\right) \\
& \quad-\Psi_{2}\left(\xi_{11}^{\star}, \xi_{33}^{\star}, w\left(b_{2}\left(\xi_{11}^{\star}, \xi_{33}^{\star}\right)\right)\right)+\delta \\
& \quad \leq \max \left\{\mid \Psi_{1}\left(\xi_{11}^{\star}, \xi_{22}^{\star}, h\left(b_{1}\left(\xi_{11}^{\star}, \xi_{22}^{\star}\right)\right)\right)\right. \\
& \quad-\Psi_{2}\left(\xi_{11}^{\star}, \xi_{22}^{\star}, w\left(b_{2}\left(\xi_{11}^{\star}, \xi_{22}^{\star}\right)\right)\right) \mid \\
& \quad \mid \Psi_{1}\left(\xi_{11}^{\star}, \xi_{33}^{\star}, h\left(b_{1}\left(\xi_{11}^{\star}, \xi_{33}^{\star}\right)\right)\right) \\
& \left.-\Psi_{2}\left(\xi_{11}^{\star}, \xi_{33}^{\star}, w\left(b_{2}\left(\xi_{11}^{\star}, \xi_{33}^{\star}\right)\right)\right) \mid\right\}+\delta .
\end{aligned}
$$

So we can write

$$
\begin{aligned}
& \left|S h\left(\xi_{11}^{\star}\right)-T w\left(\xi_{11}^{\star}\right)\right| \\
& \quad<\max \left\{\mid \Psi_{1}\left(\xi_{11}^{\star}, \xi_{22}^{\star}, h\left(b_{1}\left(\xi_{11}^{\star}, \xi_{22}^{\star}\right)\right)\right)\right. \\
& \quad-\Psi_{2}\left(\xi_{11}^{\star}, \xi_{22}^{\star}, w\left(b_{2}\left(\xi_{11}^{\star}, \xi_{22}^{\star}\right)\right)\right) \mid \\
& \quad \mid \Psi_{1}\left(\xi_{11}^{\star}, \xi_{33}^{\star}, h\left(b_{1}\left(\xi_{11}^{\star}, \xi_{33}^{\star}\right)\right)\right) \\
& \left.\quad-\Psi_{2}\left(\xi_{11}^{\star}, \xi_{33}^{\star}, w\left(b_{2}\left(\xi_{11}^{\star}, \xi_{33}^{\star}\right)\right)\right) \mid\right\}+\delta \\
& \quad=\max \left\{\mid \Psi_{1}\left(\xi_{11}^{\star}, \xi_{22}^{\star}, h\left(b_{1}\left(\xi_{11}^{\star}, \xi_{22}^{\star}\right)\right)\right)\right. \\
& \quad-\Psi_{2}\left(\xi_{11}^{\star}, \xi_{22}^{\star}, w\left(b_{2}\left(\xi_{11}^{\star}, \xi_{22}^{\star}\right)\right)\right) \mid+\delta \\
& \mid \Psi_{1}\left(\xi_{11}^{\star}, \xi_{33}^{\star}, h\left(b_{1}\left(\xi_{11}^{\star}, \xi_{33}^{\star}\right)\right)\right) \\
& \left.\quad-\Psi_{2}\left(\xi_{11}^{\star}, \xi_{33}^{\star}, w\left(b_{2}\left(\xi_{11}^{\star}, \xi_{33}^{\star}\right)\right)\right) \mid+\delta\right\} \\
& \left|S h\left(\xi_{11}^{\star}\right)-T w\left(\xi_{11}^{\star}\right)\right|<\max \{|P-Q|+\delta,|R-S|+\delta\},
\end{aligned}
$$

where $P=\Psi_{1}\left(\xi_{11}^{\star}, \xi_{22}^{\star}, h\left(b_{1}\left(\xi_{11}^{\star}, \xi_{22}^{\star}\right)\right)\right), Q=\Psi_{2}\left(\xi_{11}^{\star}, \xi_{22}^{\star}\right.$, $\left.w\left(b_{2}\left(\xi_{11}^{\star}, \xi_{22}^{\star}\right)\right)\right), R=\Psi_{1}\left(\xi_{11}^{\star}, \xi_{33}^{\star}, h\left(b_{1}\left(\xi_{11}^{\star}, \xi_{33}^{\star}\right)\right)\right)$, and $S=$ $\Psi_{2}\left(\xi_{11}^{\star}, \xi_{33}^{\star}, w\left(b_{2}\left(\xi_{11}^{\star}, \xi_{33}^{\star}\right)\right)\right)$.

Similarly, one can obtain (66), if opt ${ }_{\xi_{22}^{\star} \in \widetilde{F}}=\sup _{\xi_{22}^{\star} \in \widetilde{F}}$. Now, with the help of (66), we have

$$
\begin{aligned}
& \left|S h\left(\xi_{11}^{\star}\right)-T w\left(\xi_{11}^{\star}\right)\right| \leq \max \{|P-Q|+\delta,|R-S|+\delta\} \\
& \quad \leq \max \{\Theta(h, w)+\delta, \Theta(h, w)+\delta\}
\end{aligned}
$$

where $\left(\xi_{11}^{\star}, h, w\right) \in \widetilde{E} \times B(\widetilde{E}) \times B(\widetilde{E})$.

Since $\delta$ is arbitrary one can get

$$
\|S h-T w\| \leq \Theta(h, w), \quad \forall h, w \in B(\widetilde{E}) .
$$

By taking $\psi(\tau)=\tau, \phi(\tau)=0$ and $p=1$ in Theorem 13 we get (68). Hence, we deduce that the mappings $S, T, f$, and $g$ have a common fixed point in $B(\widetilde{E})$; that is, system (54) has a solution.

\section{Competing Interests}

The authors declare that they have no competing interests.

\section{Authors' Contributions}

All authors read and approved the final version.

\section{References}

[1] L. Gholizadeh, "A fixed point theorem in generalized ordered metrice spaces with application," The Journal of Nonlinear Science and Applications, vol. 6, pp. 244-251, 2013.

[2] A. Pansuwan, W. Sintunavarat, V. Parvaneh, and Y. J. Cho, "Some fixed point theorems for $(\alpha, \theta, k)$-contraction multivalued mappings with some applications," Fixed Point Theory and Applications, vol. 2015, article 132, 2015.

[3] S. Banach, "Sur les opérations dans les ensembles abstraits et leur application aux équations intégrales," Fundamenta Mathematicae, vol. 3, no. 1, pp. 133-181, 1922.

[4] Ya. I. Alber and S. Guerre-Delabriere, "Principle of weakly contractive maps in hilbert spaces," in New Results in Operator Theory and Its Applications: The Israel M. Glazman Memorial Volume, vol. 98 of Operator Theory: Advances and Applications, pp. 7-22, Springer, Berlin, Germany, 1997.

[5] D. S. Jaggi, "Some unique fixed point theorems," Indian Journal of Pure and Applied Mathematics, vol. 8, no. 2, pp. 223-230, 1977.

[6] S. B. Nadler Jr., "Multi-valued contraction mappings," Pacific Journal of Mathematics, vol. 30, pp. 475-488, 1969.

[7] M. A. Kutbi and W. Sintunavarat, "Fixed point theorems for generalized $\omega_{\alpha}$-contraction multivalued mappings in $\alpha \alpha$ complete metric spaces," Fixed Point Theory and Applications, vol. 2014, article 139, 2014.

[8] M. Shen and S. H. Hong, "Common fixed points for generalized contractive multivalued operators in complete metric spaces," Applied Mathematics Letters, vol. 22, no. 12, pp. 1864-1869, 2009.

[9] B. E. Rhoades, "Some theorems on weakly contractive maps," Nonlinear Analysis, vol. 47, no. 4, pp. 2683-2693, 2001.

[10] P. N. Dutta and B. S. Choudhury, "A generalisation of contraction principle in metric spaces," Fixed Point Theory and Applications, vol. 2008, Article ID 406368, 2008.

[11] M. A. Kutbi and W. Sintunavarat, "On new fixed point results for $(\alpha, \psi, \xi)$-contractive multi-valued mappings on $\alpha$-complete metric spaces and theirconsequences," Fixed Point Theory and Applications, vol. 2015, article 2, 2015.

[12] Q. Zhang and Y. Song, "Fixed point theory for generalized $\phi$ weak contractions," Applied Mathematics Letters, vol. 22, no. 1, pp. 75-78, 2009.

[13] D. Đorić, "Common fixed point for generalized $(\psi, \emptyset)$-weak contractions," Applied Mathematics Letters, vol. 22, no. 12, pp. 1896-1900, 2009.

[14] G. Jungck, "Commuting mappings and fixed points," The American Mathematical Monthly, vol. 83, no. 4, pp. 261-263, 1976. 
[15] G. Jungck, "Compatible mappings and common fixed points," International Journal of Mathematics and Mathematical Sciences, vol. 9, no. 4, pp. 771-779, 1986.

[16] G. Jungck and B. E. Rhoades, "Fixed points for set valued functions without continuity," Indian Journal of Pure and Applied Mathematics, vol. 29, no. 3, pp. 227-238, 1998.

[17] M. A. Al-Thagafi and N. Shahzad, "A note on occasionally weakly compatible maps," International Journal of Mathematical Analysis, vol. 3, no. 1-4, pp. 55-58, 2009.

[18] M. S. Khan, "Common fixed point theorems for multivalued mappings," Pacific Journal of Mathematics, vol. 95, no. 2, pp. 337347, 1981.

[19] S. L. Singh, K. S. Ha, and Y. J. Cho, "Coincidence and fixed points of nonlinear hybrid contractions," International Journal of Mathematics and Mathematical Sciences, vol. 12, no. 2, pp. 247-256, 1989.

[20] S. L. Singh and S. N. Mishra, "Nonlinear hybrid contractions," Journal of Natural \& Physical Sciences, vol. 5-8, pp. 191-206, 1991-1994.

[21] A. Aliouche and V. Popa, "General common fixed point theorems for occasionally weakly compatible hybrid mappings and applications," Novi Sad Journal of Mathematics, vol. 39, no. 1, pp. 89-109, 2009.

[22] H. Kaneko and S. Sessa, "Fixed point theorems for compatible multi-valued and single-valued mappings," International Journal of Mathematics and Mathematical Sciences, vol. 12, no. 2, pp. 257-262, 1989.

[23] M. Abbas and B. E. Rhoades, "Common fixed point theorems for hybrid pairs of occasionally weakly compatible mappings satisfying generalized contractive condition of integral type," Fixed Point Theory and Applications, vol. 2007, Article ID 054101, 2007.

[24] M. Aamri and D. El Moutawakil, "Some new common fixed point theorems under strict contractive conditions," Journal of Mathematical Analysis and Applications, vol. 270, no. 1, pp. 181$188,2002$.

[25] W. Sintunavarat and P. Kumam, "Common fixed point theorems for a pair of weakly compatible mappings in fuzzy metric spaces," Journal of Applied Mathematics, vol. 2011, Article ID 637958, 14 pages, 2011.

[26] A. A. N. Abdou, "Common fixed point theorems for hybrid contractive pairs with the (CLR)-property," Fixed Point Theory and Applications, vol. 2015, article 138, 2015.

[27] A. A. Abdou, "Common fixed point results for multi-valued mappings with some examples," Journal of Nonlinear Science and Its Applications, vol. 9, no. 3, pp. 787-798, 2016.

[28] A. Roldán and W. Sintunavarat, "Common fixed point theorems in fuzzy metric spaces using the CLRg property," Fuzzy Sets and Systems, vol. 282, pp. 131-142, 2016.

[29] Z.-q. Liu and S. M. Kang, "Existence and uniqueness of solutions for two classes of functional equations arising in dynamic programming," Acta Mathematicae Applicatae Sinica. English Series, vol. 23, no. 2, pp. 195-208, 2007. 


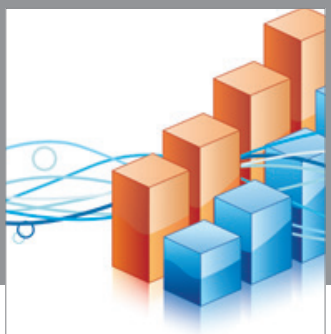

Advances in

Operations Research

vatem alat4

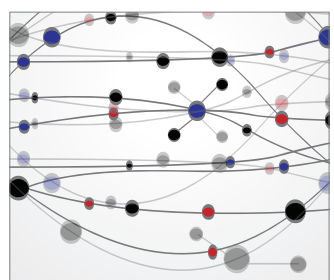

\section{The Scientific} World Journal
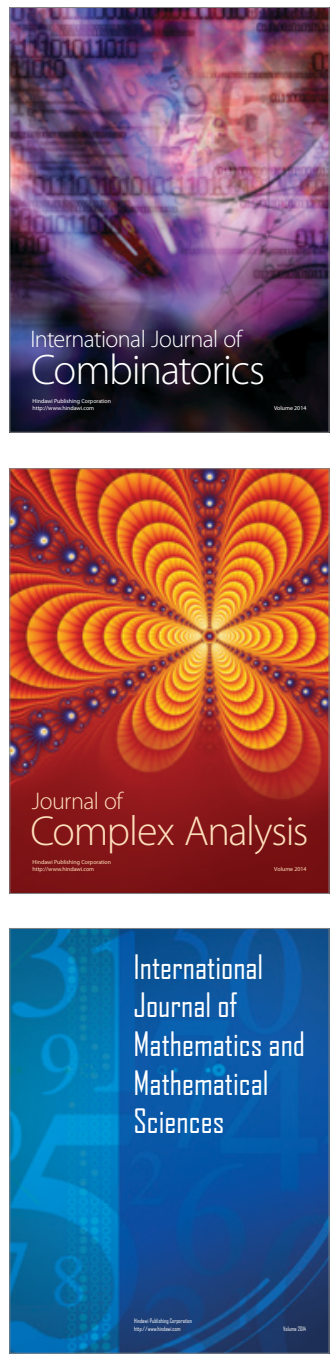
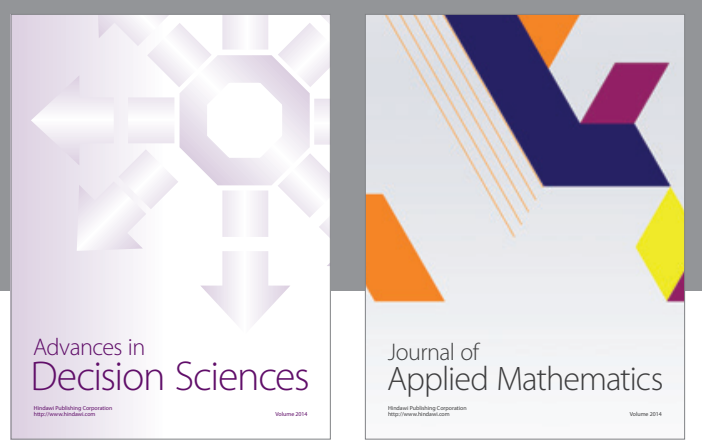

Algebra

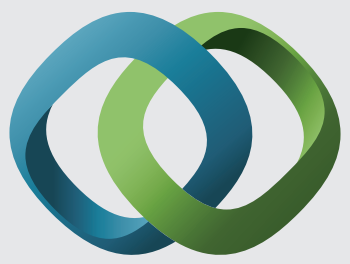

\section{Hindawi}

Submit your manuscripts at

http://www.hindawi.com
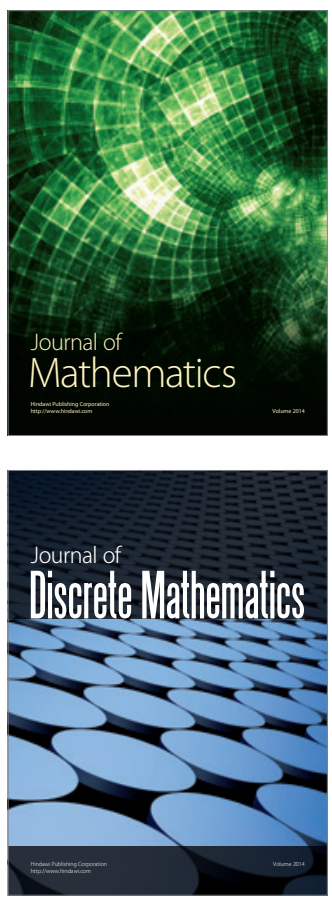

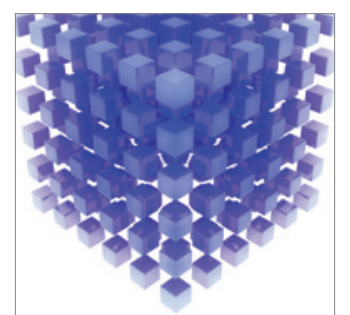

Mathematical Problems in Engineering
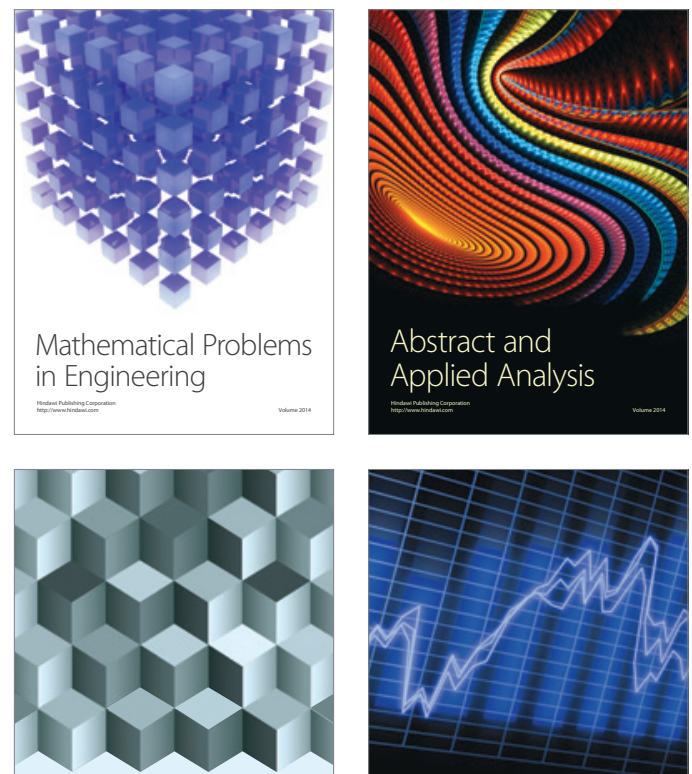

Journal of

Function Spaces

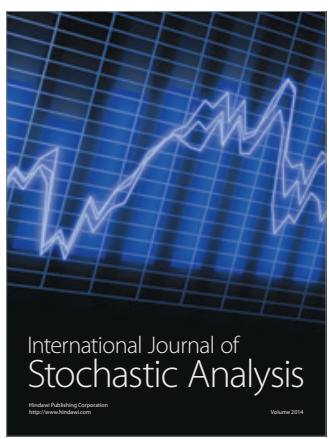

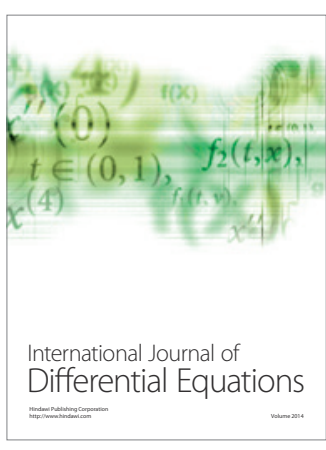
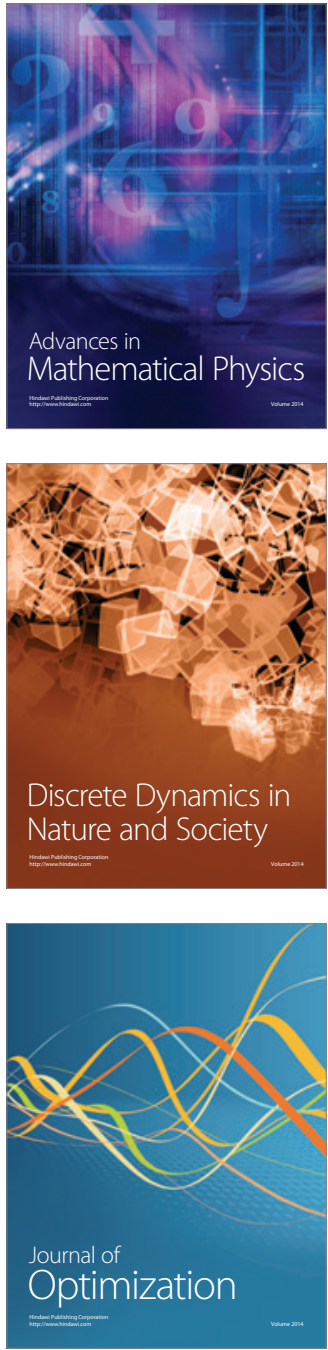